\title{
论 文群体智能及工程应用专辑
}

\section{一种基于自适应种群变异鸽群优化的航天器集群 轨道规划方法}

华冰 ${ }^{*}$, 刘禼鹏, 孙胜刚, 吴云华, 陈志明

南京航空航天大学航天学院, 南京 210016

*E-mail: huabing@nuaa.edu.cn

收稿日期: 2019-08-15; 接受日期: 2019-10-14; 网络版发表日期: 2020-04-03

北京航空航天大学虚拟现实技术与系统国家重点实验室项目(编号：VRLA2018A01)和国家自然科学基金项目(批准号：61973153，61673208) 资助

摘要航天器集群在复杂条件下的轨道规划问题是当前航天领域的热点以及难点. 本文针对分布式集群航天器 在队形变换过程中的轨道最优规划问题进行了研究，提出了基于自适应种群变异的鸽群算法(adaptive population variation pigeon-inspired optimization, APVPIO). 本文对经典PIO算法中的核心演化算法、演化停滞以及易陷入局 部最优解问题进行了研究. 同时针对经典PIO算法的适应度函数进行了研究，并且结合轨道规划问题进行了改进. 最后基于自适应种群变异的鸽群算法进行了仿真实验，结果表明，APVPIO算法，相比于经典PIO算法、PSO算法 在极大减少计算量的同时, 有更优规划结果、更深的种群演化深度以及更快的收敛速度, 可以满足航天器集群在 复杂约束条件下的轨道规划问题.

关键词轨道规划, PIO算法, 航天器避障, 算法种群演化, 航天器集群

\section{1 引言}

随着空间任务的复杂化以及航天器小型化技术的 日益成熟，小型航天器集群化已成为当前空间技术未 来的发展方向，成为各航天大国争先抢占的技术制 高点.

小型航天器集群在各成员星协同工作和集群队形 重构时, 最为重要的是要解决卫星各成员星之间的避 障问题，以及变轨燃料消耗的最优化问题. 针对该问 题Kala等人 ${ }^{[1]}$ 和Elshamli等人 ${ }^{[2]}$ 分别使用了蚁群算法以 及遗传算法生成了路径近似最优解，但存在算法收敛
速度慢, 易陷入局部最优解的问题. Warre ${ }^{[3]}$ 使用的人 工势场法完成了在空间三维环境下的路径最优规划, 但是势场法本身存在局部最小点, 并且随着优化环境 复杂度的提高, 算法的使用将受到限制. Carroll等人 ${ }^{[4]}$ 提出了 $\mathrm{A}$ *算法可以满足高维度的优化问题, 但是随着 维度的提高, 算法的时空要求将很难得到满足.

北京航空航天大学的霍梦真和段海滨 ${ }^{[5]}$ 为了优化 无人机目标搜索方法, 提出一种基于Agent路由和光传 感器的自适应变异多目标鸽群优化算法. 利用自适应 飞行机制可以获得较好的鸽群分布，种群具有多样性 和收玫性. 并且利用变异机制简化了鸽群优化算法中

引用格式: 华冰, 刘睿鹏, 孙胜刚, 等. 一种基于自适应种群变异鸽群优化的航天器集群轨道规划方法. 中国科学: 技术科学, 2020, 50: 453-460 Hua B, Liu R P, Sun S G, et al. Spacecraft cluster orbit planning method based on adaptive population mutated pigeon group optimization (in Chinese). Sci Sin Tech, 2020, 50: 453-460, doi: 10.1360/SST-2019-0285 
的模型, 提高了搜索效率.

本文针对航天器轨道在复杂约束条件下的规划问 题进行了研究. 针对经典PIO算法的核心迭代算法、 演化策略以及适应度函数进行对应的改进以及优化, 提出了基于自适应种群变异的鸽群算法(adaptive population variation pigeon-inspired optimization, APVPIO)进行轨道规划. 降低了规划算法计算量, 并且解决 了传统算法易陷入局部最优解、种群演化停滞等问 题, 实现对轨道的最优控制.

\section{2 问题模型构建}

在工作过程中, 航天器集群经常需要依据观测任 务的不同进行队形的重构, 在重构的过程中需要对相 邻航天器的运动区域进行规避以免发生碰撞. 机动航 天器在由 $\mathrm{A}$ 位置经过 $\mathrm{B}$ 位置机动到 $\mathrm{C}$ 位置期间需要对 前进过程中各个障碍航天器进行避障.

\section{1 航天器轨道机动模型}

由于航天器集群队形变化的相对运动距离相对较 近, 于是本文采用不考虑摄动力偏差的HILL方程建立 相对运动模型. 定义相对运动坐标系如下: 原点位于机 动航天器的质心, $x$ 轴为卫星到地心连线的反方向, $y$ 轴 为卫星的飞行速度方向, $z$ 轴符合右手系, 相对轨道参 考坐标系如图1所示. 障碍航天器相对机动航天器位置 的解析表达式如下所示:

$$
\left\{\begin{aligned}
x(t)= & \frac{\dot{x}_{0}}{n} \sin (n t)-\left(\frac{2 \dot{y}_{0}}{n}+3 x_{0}\right) \cos (n t) \\
& +2\left(\frac{\dot{y}_{0}}{n}+2 x_{0}\right), \\
y(t)= & 2\left(\frac{2 \dot{y}_{0}}{n}+3 x_{0}\right) \sin (n t)+\frac{2 \dot{x}_{0}}{n} \cos (n t) \\
& -3\left(\dot{y}_{0}+2 n x_{0}\right) t+\left(y_{0}-\frac{2 x_{0}}{n}\right), \\
z(t)= & \frac{\dot{z}_{0}}{n} \sin (n t)+z_{0} \cos (n t),
\end{aligned}\right.
$$

其中, $n=\sqrt{\frac{\mu}{r^{3}}}$. 由上式以及航天器的尺寸可以得知在 $t$ 时间后障碍航天器的位置以及避障区域的大小.

\section{2 轨道规划模型建立}

在实际工程中, 航天器轨道控制发动机的喷气速

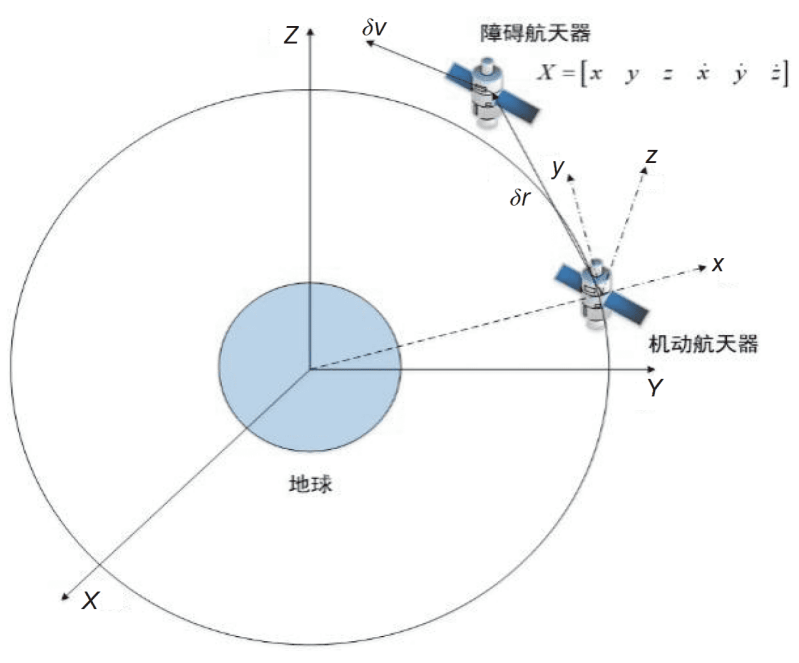

图 1 (网络版彩图)相对轨道参考坐标系

Figure 1 (Color online) Relative orbital reference coordinate system.

度是有限的，在算法中体现为将喷气速度控制在一定 的范围内.

喷气速度约束:

$\left|v_{i}\right| \leq \lambda_{v}$,

其中, $v_{i}$ 为控制节点喷气速度大小, $\lambda_{v}$ 为航天器最大喷 气速度. 为了避免航天器在机动过程中与集群中其他 航天器发生碰撞, 设定路径约束:

$\left|\left[x_{b}(t), y_{b}(t), z_{b}(t)\right]-\left[x_{o}(t), y_{o}(t), z_{o}(t)\right]\right| \geq R$.

于是可以归纳成以下的形式:

$\min : J=$ fitness $(x, y, z)$, (性能指标),

s.t.

$\begin{cases}-\lambda_{v} \leq v_{i} \leq \lambda_{v}, i=1,2,3, & \text { (喷气速度边界约束), } \\ \left|X_{b}-X_{o}\right| \geq R, & \text { (路径约束), } \\ X\left(t_{0}\right)=X_{0}, & \text { (初始条件), } \\ X\left(t_{f}\right)=X_{f}, & \text { (终端条件). }\end{cases}$

$J$ 的具体表现形式如式(16)所示. 代价函数(4)中的 4个约束依次代表喷气速度边界约束、路径约束、初 始条件和终端条件.

\section{3 基于自适应种群变异的鸽群算法}

在当前, 各种种群遗传算法发展迅速, 并且在解决 各类优化问题中均发挥着重要的作用. 其中最为典型 的有粒子群算法 $(\mathrm{PSO}) 、$ 、蜂群优化算法 $(\mathrm{ABC})$ 、水滴 
优化算法(IWD)以及 $A$ *算法等. 种群遗传算法具有算 法参数设置简单、应用领域广泛等特点, 但是随着优 化模型的复杂，算法易早熟收玫、易陷入局部最优解 和计算量大等问题逐渐突出.

PIO算法是Duan ${ }^{[6 \sim 9]}$ 受到鸽群在归巢中的导航过 程启发发展而来, 算法创新性地使用了两段式的演化 算子, 分别对应远离目标时的地图指南针算子和接近 目标时的地标算子.

\section{1 适应种群变异的鸽群改进算法}

虽然经典款PIO算法在传统群类算法的缺点方面 有了一定的改善, 但是依然存在算法计算量较大, 易陷 入局部最优解、种群演化停滞、优化结果平滑度较低 等问题 ${ }^{10]}$. 这些问题使得航天器或者其他执行机构频 繁启动, 以跟随指令, 使系统发生抖动 ${ }^{[11 ~ 13]}$, 在消耗燃 料的同时对机械结构的磨损也较大, 影响航天器的使 用寿命.

本文对鸽群算法种群迭代演化以及适应度函数两 个方面进行了重点研究, 并针对现有遗传算法的不足, 在种群演化阶段提出了基于“搜寻”, “变异”的鸽群动 态寻优策略. 以解决现有算法在收玫速度、局部最 优、演化深度方面的问题.

鸽群动态“搜寻”, 是在种群完成初始化后即开始 迭代对整个空间进行搜索寻优. 本节针对经典PIO算 法的核心迭代算子进行了研究, 全局最优个体的位置 信息是种群在演化过程中的主要参考.

在迭代初期种群进化并不深入, 全局最优个体相 对普通个体并不具有十分明显的优势, 从而对整个种 群的参考价值并不高, 而经典PIO算法在速度更新时 全局最优解的权值为一个随机数, 这使得全局最优个 体并不能很好地指导种群的演化.

本文针对全局最优个体加入了全局更新算子 $C_{g}$, 使全局最优个体只有在优势明显时才具有更高的权 值, 而迭代初期的权值较小.

在研究中发现, 在迭代初期单个个体的演化经验 相比全局最优个体具有更高的参考价值, 可以达到帮 助收玫的目的. 而经典PIO算法并没有对该信息加以 利用, 于是本文创新性地引入了自适应算子 $C_{p}$, 使之 可依据种群中个体当前迭代状态与前两次迭代的演化 趋势进行自适应调整, 在鸽群进化缓慢时增大搜索的
范围, 以达到动态最优搜索的目的.

自适应改进型地图指南针的优化算子更新如下:

$$
\begin{aligned}
& V_{i}(t+1)=w \cdot V(t)+C_{p}\left[X_{p}(t)-X_{i}(t)\right] \\
& +C_{g}\left[X_{g}-X_{i}(t)\right] \\
& X(t+1)=X(t)+V(t+1), \\
& C_{g}=\log _{T_{\max }}^{t}, \\
& C_{p}=\mathrm{e}^{\left[\frac{\text { fitness }(t-3)}{\mathrm{fitness}(t-2)}-\frac{\text { fitness }(t-2)}{\mathrm{fitness}(t-1)}\right]}, \\
& w=w_{\max }-\frac{\left(w_{\max }-w_{\min }\right) \cdot t}{T_{\max }},
\end{aligned}
$$

其中, $V_{i}(t)$ 为第 $i$ 只个体在 $t$ 次迭代中的速度, 同时满足 控制指标中的限制性约束条件，若在迭代中超过限制 性条件, 该速度取航天器喷气速度的最大值. $X_{i}(t)$ 为第 $i$ 只个体在 $t$ 次迭代中的位置, $X_{p}$ 为第 $i$ 只个体在 $t$ 次迭代 中的局部最优位置, $X_{g}$ 为所有个体在 $t$ 次迭代中的全局 最优位置, fitness $(t)$ 为第 $i$ 只个体在第 $t$ 次迭代中的适应 度值, $w$ 为惯性因子, $w_{\max }$ 为惯性因子最大值, 此处取 $0.9, w_{\text {min }}$ 为惯性因子最小值, 此处取 $0.4, T_{\text {max }}$ 为最大迭 代次数, $t$ 为当前迭代次数.

局部最优解问题是经典PIO算法以及同类遗传算 法所共同存在的. 如何抑制算法陷入局部最优解, 以及 在算法陷入局部最优解后如何脱离局部最优解, 是解 决此类问题的关键.

本文在上述优化算子的基础上进一步加入了种群 变异算子, 使鸽群中部分个体“变异”. 当种群适应度值 的变化率连续 30 次低于 0.01 时, 说明种群有可能陷入 了局部最优解. 此时对鸽群中距离当前局部最优解较 远的一半(即按适应度值排序的后一半个体)进行变异, 变异算子将产生以当前值为均值, 当前适应度值的倒 数为方差的一个随机数. 可使后一半种群在下次迭代 中被激活, 进行大范围搜索, 使种群脱离局部最优解.

由于仅对后一半种群进行变异, 即使算法当前已 找到全局最优解，大范围搜索的结果不会优于当前已 找到的全局最优解, 故算法仍是收玫的.

最终地图指南针的优化算子如下:

$$
\begin{aligned}
V(t+1)= & w \cdot V(t)+C_{p}\left[X_{p}-X_{i}(t)\right]+C_{g}\left[X_{g}-X_{i}(t)\right] \\
& +C_{r}\left[R_{d}(t+1)-X_{i}(t)\right],
\end{aligned}
$$


$C_{r}= \begin{cases}0, & \text { others, } \\ 1, & \frac{\mathrm{dfitness}(t) / \mathrm{d} t<0.1}{\text { fitness }(t) \text { atlatrer half of population }},\end{cases}$

$R_{d}(t+1)=N\left(X_{i}(t), \frac{\mathrm{d} t}{\operatorname{dfitness}(t)}\right)$,

其中, $C_{r}$ 为激活因子, $R_{d}(t+1)$ 为以 $X_{i}(t)$ 为均值, $\frac{\mathrm{d} t}{\mathrm{dfitness}(t)}$ 为方差的一个随机数.

\section{2 基于自适应种群变异的鸽群改进型算法}

在经典PIO算法的适应度函数中，仅对规划路径 的长短以及与障碍区域的距离做了定义．而在实际问 题中，非威胁区域的路径规划依然对航天器的整体机 动最优化程度有着重要的影响 ${ }^{[14]}$.

经典PIO算法的适应度函数仅将距离与威胁程度 作为评价个体优劣的参考，在计算与威胁中心的距离 时是将姿态演化等分为若干小段，每个小段到威胁中 心的距离作为是否构成威胁的判定标准. 第 $i$ 只个体的 适应度函数表达式为

$$
\begin{aligned}
& \text { fitness }(i)=w_{1} \cdot \operatorname{distance}(i)+w_{2} \cdot \operatorname{threat}(i), \\
& \text { diatance }(i)=\sum_{j=0}^{n} L_{i, j},
\end{aligned}
$$

$$
\operatorname{threat}(i)=\left\{\begin{aligned}
& 0, \text { if } R_{i}>R_{j}, \\
& \frac{L_{i, j}}{5} \sum_{k=1}^{N_{t}} t_{k}\left(\frac{1}{d_{0.1, k}^{4}}+\frac{1}{d_{0.3, k}^{4}}\right. \\
&\left.\quad+\frac{1}{d_{0.5, k}^{4}}+\frac{1}{d_{0.7, k}^{4}}+\frac{1}{d_{0.9, k}^{4}}\right), \text { esle, }
\end{aligned}\right.
$$

其中, $w_{1}$ 与 $w_{2}$ 分别为权重因子, 用于平衡鸽群优先避障
或优先姿态调整时间最短, 且 $w_{1}+w_{2}=1, L_{i, j}$ 为第 $i$ 只个体 的第 $j$ 个规划分段的长度, $t_{k}$ 为第 $k$ 个威胁的威胁等级, $N_{t}$ 为威胁的个数, $d_{0.1, k}$ 为该次姿态调整的 $1 / 10$ 处与第 $k$ 个威胁中心的距离, $R_{j}$ 为第 $j$ 个威胁的威胁半径, $R_{i, j}$ 为 第 $i \varepsilon$ 到第 $j$ 个威胁中心的平均距离 ${ }^{[15]}$.

在经典PIO算法中，用于定义障碍区域威胁程度 的 $t_{k}$ (见式(14))为一变量, 可针对威胁区域的不同设定 不同的威胁等级, 而航天器避障问题与飞机穿过雷达 区域不同，任何与威胁区域的接触均会引起碰撞，这 是禁止发生的. 于是本文把 $t_{k}$ 改造为与航天器同障碍 航天器距离的函数, 距离越近威胁程度越高. 并且在 远离障碍时威胁程度迅速降低, 以避免航天器不必要 的机动. 对于航天器机动问题, 优化的轨迹应尽量平 滑, 所以在适应度函数中应加入角度机动因子.

于是适应度函数改进如下式:

$$
\begin{aligned}
\text { fitness }(i)= & w_{1} \cdot \text { threat_in }(i)+w_{2} \cdot \text { threat_out }(i) \\
& +w_{1} \cdot \text { distance }(i)+w_{4} \cdot \operatorname{angle}(i),
\end{aligned}
$$

其中, $w_{1}+w_{2}+w_{3}+w_{4}=1$.

并且在规划空间中针对路径进入威胁区域内(图 2)与威胁区域外(图3)建立不同的适应度函数. 当规划 路径进入威胁区域时, 其适应度函数为

$$
\text { threat_in }(i)=\left\{\begin{aligned}
\frac{L_{i, k}}{5} t_{j} \sum_{j=1}^{M}\left(\frac{1}{d_{0.1, j}^{4}}+\frac{1}{d_{0.3, j}^{4}}+\frac{1}{d_{0.5, j}^{4}}\right. \\
\left.\quad+\frac{1}{d_{0.7, j}^{4}}+\frac{1}{d_{0.9, j}^{4}}\right),\left|X_{i}(t)-T_{j}\right|<R_{j}, \\
0, \quad \text { others. }
\end{aligned}\right.
$$

当规划路径未进入威胁区域时, 其适应度函数为

threat_out $(i)=\left\{\begin{array}{l}t_{j} \sum_{j=1}^{M}\left(\frac{1}{d_{0.1, j}^{4}-R_{j}{ }^{4}}+\frac{1}{d_{0.3, j}^{4}-R_{j}{ }^{4}}+\frac{1}{d_{0.5, j}^{4}-R_{j}{ }^{4}}+\frac{1}{d_{0.7, j}^{4}-R_{j}{ }^{4}}+\frac{1}{d_{0.9, j}^{4}-R_{j}{ }^{4}}\right),\left|X_{i}(t)-T_{j}\right|>R_{j}, \\ 0, \quad \text { other. }\end{array}\right.$

$t_{j}=\mathrm{e}^{\ln \left(R_{j}\right)-\frac{\ln \left(R_{j}\right)-1}{R_{j}} \cdot \frac{\left|\overline{T_{j} X_{i-1}} \times \overline{X_{i-1} X_{i}}\right|}{\left|X_{i-1} X_{i}\right|},}$,

其中, $T_{j}$ 为第 $j$ 个威胁区域的中心坐标, $X_{i}$ 与 $X_{i-1}$ 分别为 第 $i$ 个路径点与第 $i-1$ 个路径点的威胁等级, 其变化趋 势如图4所示 $\left(R_{j}=30\right)$.
综上所述, APVPIO算法流程如图5所示.

\section{4 仿真实验与结果分析}

实验设定机动航天器相对运动坐标系, 机动航天 器在相对运动坐标系下开始机动时位于坐标系原点 


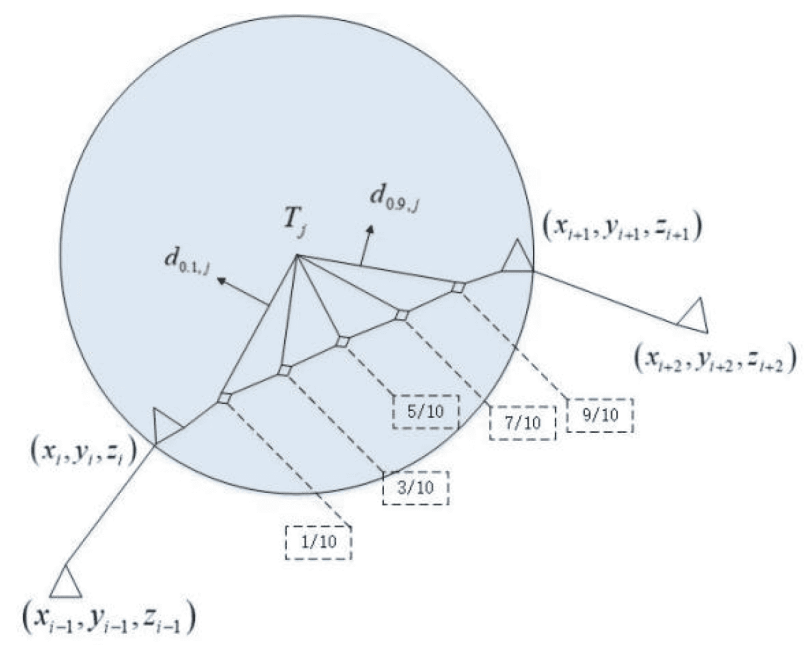

图 2 (网路版彩图)路径进入威胁区域示意图

Figure 2 (Color online) The path entering the threat zone.

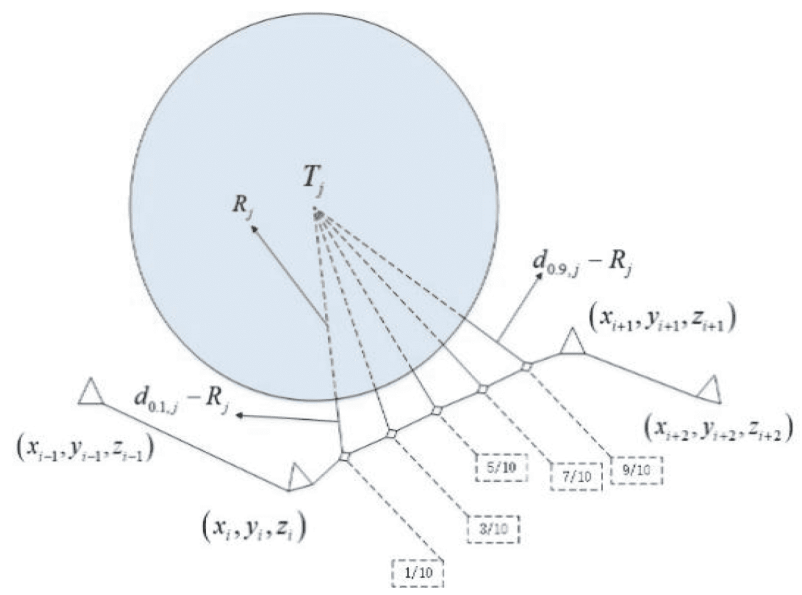

图 3 (网路版彩图)路径未进入威胁区域示意图

Figure 3 (Color online) Schematic diagram of the path not entering the threat zone.

$(0,0,0) \mathrm{km}$, 目标位置为 $(65,80,30) \mathrm{km}$, 设定变轨航天 器前方障碍航天器的相对位置为 $(45,50,5) \mathrm{km},(8,25$, 15) $\mathrm{km},(40,68,7) \mathrm{km}$.

仿真实验条件为 8 核 $2.8 \mathrm{~Hz} 、 16 \mathrm{~GB}$ 内存、64位计 算机. 在此基础上设定算法内部参数分别如下.

(1) 经典PIO算法与改进型PIO算法: 个体数量 $M=30$, 规划维度 $D=20$, 迭代次数为 200 次(地图指南针 算子 150 次, 地标算子 50 次).

(2) PSO算法: 个体数量 $M=30$, 规划维度 $D=20$, 迭 代次数为 200 次.

(3) APVPIO算法: 个体数量 $M=30$, 规划维度 $D=20$, 迭代次数为 200 次.

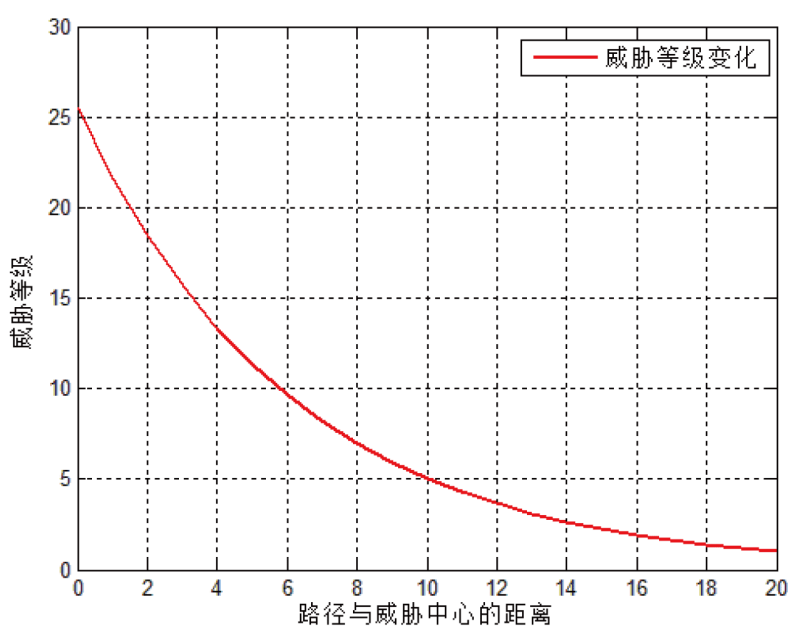

图 4 (网路版彩图)威胁等级随路径与威胁中心距离变化图 Figure 4 (Color online) Change of threat level with path and threat center distances.

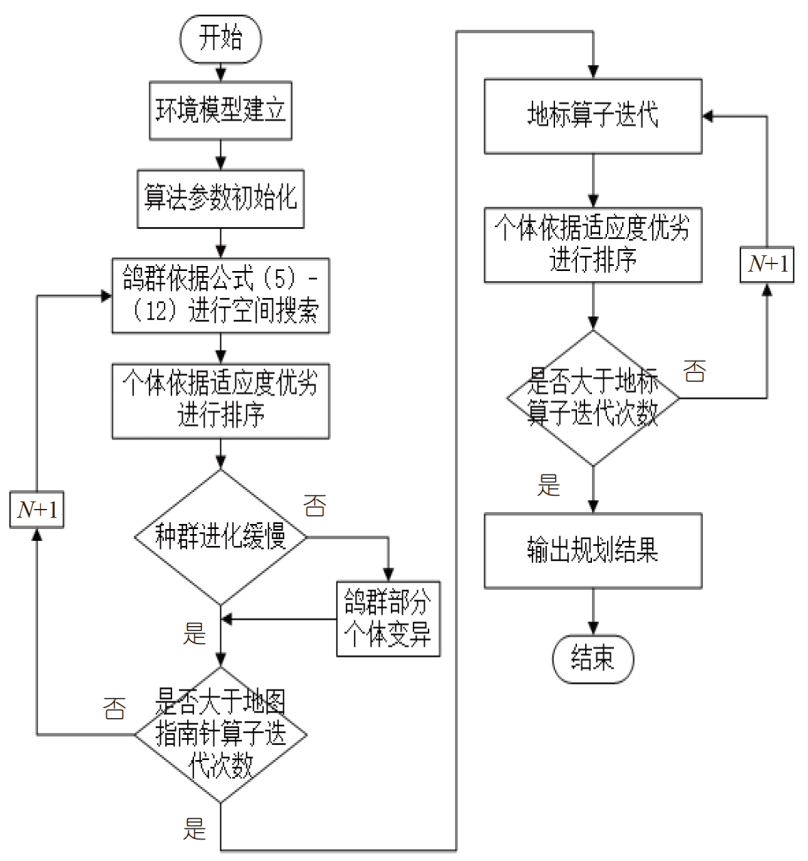

图 5 自适应种群变异的鸽群算法流程图

Figure 5 Flow chart of the APVPIO.

图6 8分别给出了在个体数量 $M=30$, 规划维度 $D=20$, 迭代次数为 200 次条件下, 经典 $\mathrm{PIO}$ 算法、 $\mathrm{PSO}$ 算法和APVPIO算法轨道规划结果的对比. 图9给出了 APVPIO算法、PIO算法、PSO算法适应度变化对比.

通过分析仿真结果发现, 在个体数量、规划维度 和迭代次数相同的情况下, APVPIO算法的路径规划 结果比PIO算法和PSO算法更加平滑且有效：在保证 


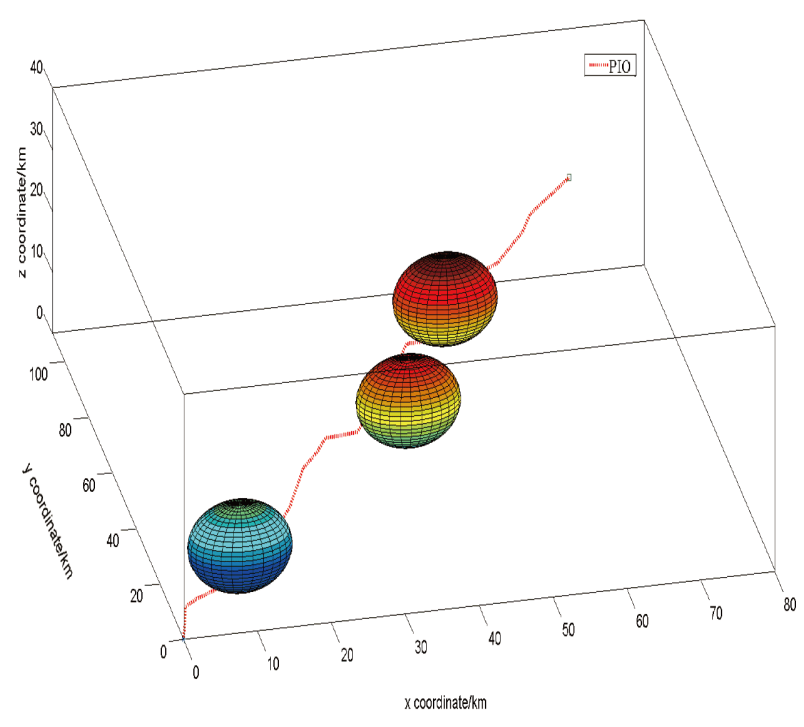

图 6 (网络版彩图)经典PIO算法轨道规划结果

Figure 6 (Color online) Orbit planning results of classic PIO.

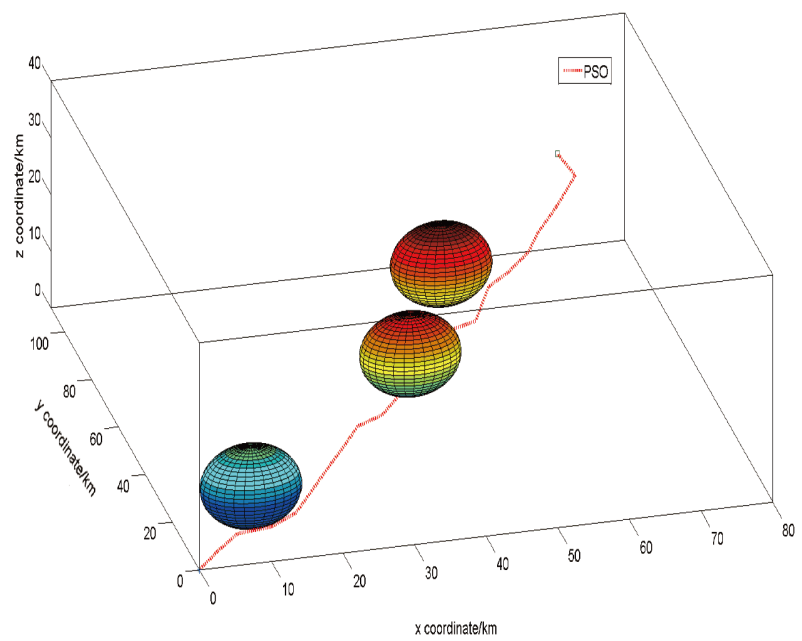

图 7 (网络版彩图)粒子群算法轨道规划结果

Figure 7 (Color online) Orbit planning results of classic PSO.

不碰撞威胁区域的同时避免了过分的机动，从而能节 省燃料. 通过分析表 1 , 从适应度变化速度来看, APVPIO算法适应度函数变化最迅速，并且更加频繁地调 用适应度函数，从而增加了种群变异演化次数，有效 避免了种群陷入局部最优．从计算时间来看，在相同 条件下，本文提出的APVPIO算法大大缩短了计算时 间, 仅为PIO算法的 $28 \%$, PSO算法的 $31 \%$.

结果表明，本文提出的APVPIO算法在极大减少 计算量的同时，有更优规划结果、更深的种群演化深

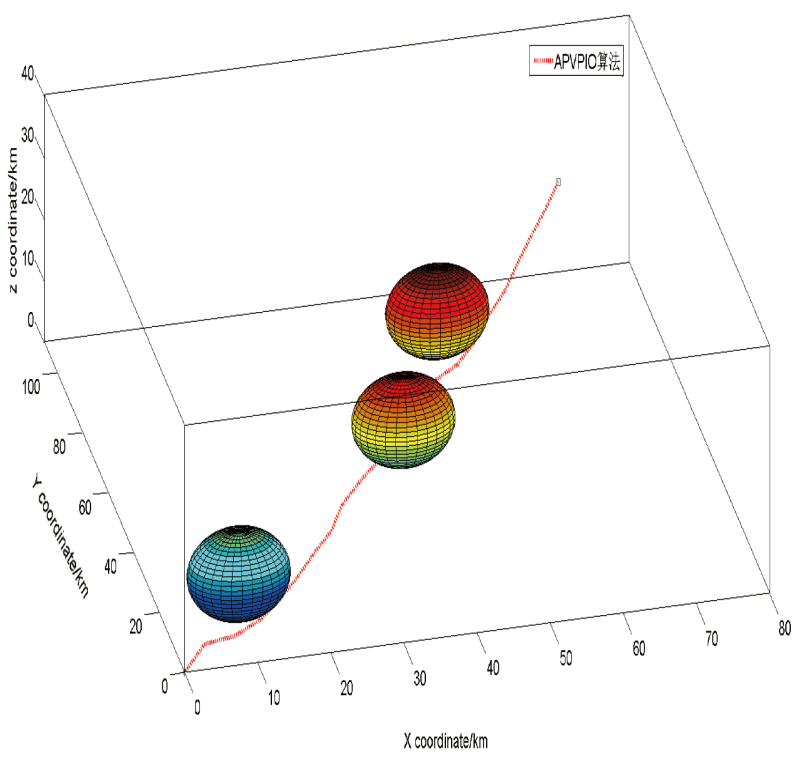

图 8 (网络版彩图)自适应种群变异的鸽群算法轨道规划结 果

Figure 8 (Color online) Orbit planning results of APVPIO.

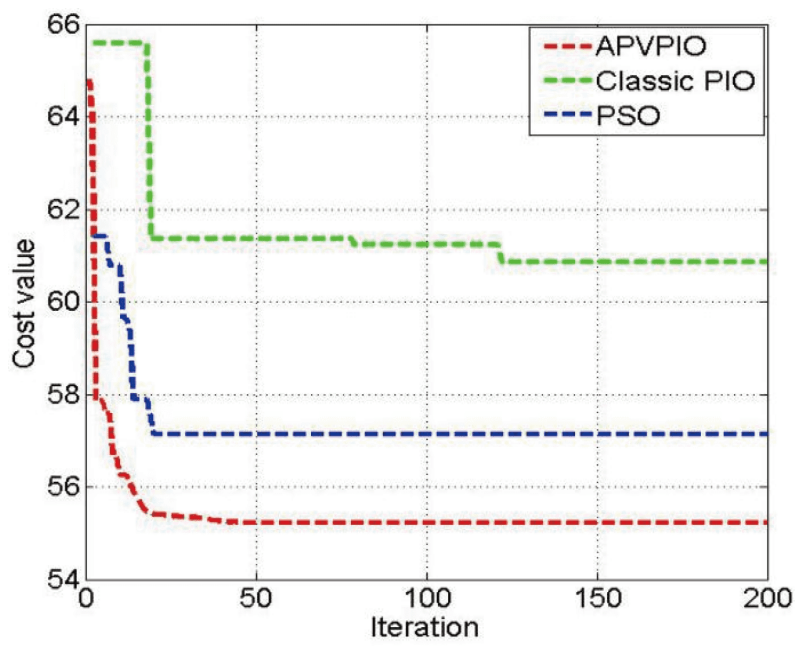

图 9 (网络版彩图)APVPIO算法、PIO算法、PSO算法适应 度变化对比

Figure 9 (Color online) Comparison of adaptability of APVPIO, PIO and PSO.

度以及更快的收玫速度, 可以满足航天器集群在复杂 约束条件下的轨道规划问题.

\section{5 结论}

本文针对航天器在复杂约束下的轨道规划问题设 计了一种基于自适应种群变异的鸽群算法. 通过对比 
表 1 APVPIO, PIO, PSO算法计算量对比

Table 1 Comparison of APVPIO, PIO, and PSO calculations

\begin{tabular}{ccccccc}
\hline 算法/调用 & 主程序调用次数 & 核心算法调用次数 & 适应度函数调用次数 & 距离函数调用次数 & 叉乘次数 & 算法总时间 $(\mathrm{s})$ \\
\hline APVPIO & 1 & 1 & 13824 & 868234 & 0 & 15.766 \\
PIO & 1 & 1 & 10463 & 1354421 & 659169 & 55.265 \\
PSO & 1 & 1 & 12088 & 1223137 & 761544 & 50.095 \\
\hline
\end{tabular}

实验可以看出本文的算法(APVPIO算法)相比于经典 PIO算法以及PSO算法, 在规划结果上更加平滑, 可减 少不必要的姿轨调整; 仿真结果表明, APVPIO算法相 比PIO以及PSO算法，种群进化更加深入，其适应度下
降值在10左右, 而其余两种算法均在5左右, 同时APVPIO算法具有更快的收敛速度; 并且通过计算量的对 比可以看出本文的改进型算法大大降低了计算量, 实 现了航天器轨道的最优规划.

\section{参考文献}

1 Kala R, Shukla A, Tiwari R. Robotic path planning in static environment using hierarchical multi-neuron heuristic search and probability based fitness. Neurocomputing, 2011, 74: 2314-2335

2 Elshamli A, Abdullah H A, Areibi S. Genetic algorithm for dynamic path planning. In: Canadian Conference on Electrical and Computer Engineering, 2004. Niagara Falls: IEEE, 2004

3 Warren C W. A technique for autonomous underwater vehicle route planning. IEEE J Ocean Eng, 1990, 15: 199-204

4 Carroll K P, Mcclaran S R, Nelson E L, et al. AUV path planning: An A* approach to path planning with consideration of variable vehicle speeds and multiple, overlapping, time-dependent exclusion zones. In: Proceedings of the 1992 Symposium on Autonomous Underwater Vehicle Technology, 1992. AUV'92. Washington, DC: IEEE, 2002. 79-84

5 Huo M Z, Duan H B. An adaptive mutant multi-objective pigeon-inspired optimization for unmanned aerial vehicle target search problem. Control Theory Appl, 1-9 [2019-09-11]

6 Duan H B, Qiao P. Pigeon-inspired optimization: A new swarm intelligence optimizer for air robot path planning. Int Jnl Intel Comp Cyber, 2014, 7: $24-37$

7 Duan H B, Qiu H X, Fan Y M. Unmanned aerial vehicle close formation cooperative control based on predatory escaping pigeon-inspired optimization. Sci Sin Tech, 2015, 45: 559-572 [段海滨, 邱华金视范铭. 基于捕食逃逸鸽群优化的无人机紧密编队协同控制. 中国科学: 技 术科学, 2015, 45: 559-572]

8 Zhang B, Duan H B. Predator-prey pigeon-inspired optimization for UAV three-dimensional path planning. In: Tan Y, Shi Y, Coello C A C, eds. Advances in Swarm Intelligence. ICSI 2014. Lecture Notes in Computer Science, vol 8795. Cham: Springer, 2014. 96-105

9 Li C, Duan H B. Target detection approach for UAVs via improved pigeon-inspired optimization and edge potential function. Aerosp Sci Tech, 2014, 39: 352-360

10 Zhou Y P. A pigeon inspired algorithm for function optimization problems (in Chinese). Dissertation for Master's Degree. Changchun: Northeast Normal University, 2016 [周雨鹏. 基于鸽群算法的函数优化问题求解. 硕士学位论文. 长春: 东北师范大学, 2016]

11 Hua B, Liu R P, Wu Y H, et al. Intelligent attitude planning algorithm based on the characteristics of low radar cross section characteristics of microsatellites under complex constraints. P I Mech Eng G-J Aerosp Eng, 2019, 233: 4-21

12 Xu R, Wang H, Xu W, et al. Rotational-path decomposition based recursive planning for spacecraft attitude reorientation. Acta Astronaut, 2018, 143: $212-220$

13 Chen X, Lu Y, Yao K, et al. Dynamic modeling and adaptive fast nonsingular terminal sliding mode control for satellite with double rotary payloads. Trans Nanjing Univ Aeronaut Astron, 2016, 33: 310-318

14 Wang L, Li M, Tang D B, et al. Dynamic path planning for mobile robot based on improved genetic algorithm (in Chinese). J Nanjing Univ Aeronaut Astron, 2016, 48: 841-846 [王雷, 李明, 唐敦兵, 等. 基于改进遗传算法的机器人动态路径规划. 南京航空航天大学学报, 2016, 48: $841-846]$ 


\title{
Spacecraft cluster orbit planning method based on adaptive population mutated pigeon group optimization
}

\author{
HUA Bing, LIU RuiPeng, SUN ShengGang, WU YunHua \& CHEN ZhiMing \\ College of Aerospace Engineering, Nanjing University of Aeronautics and Astronautics, Nanjing 210016, China
}

The orbit planning problem of spacecraft clusters under complex conditions is a hotspot and a difficult goal in the current aerospace field. This paper studies the orbital optimal programming problem of distributed cluster spacecraft in the process of formation transformation and proposes the population evolution algorithm adaptive population variation pigeon-inspired optimization (APVPIO). Based on the core evolutionary algorithm and evolutionary stagnation, there is a high tendency to fall into the local optimal solution in the classical PIO algorithm. The fitness function of the classical PIO algorithm is studied and improved with the orbit planning problem. Finally, the simulation based on the adaptive population variation algorithm is performed. The results reveal that the APVPIO algorithm has better planning results, deeper population evolution depth, and a faster convergence speed in comparison to the classical PIO algorithm and particle swarm algorithm (PSO) algorithm. Hence, it has the potential to meet the complexity requirement of spacecraft clusters and orbital planning problems.

orbit planning, PIO algorithm, spacecraft obstacle avoidance, algorithm population evolution, spacecraft cluster doi: 10.1360/SST-2019-0285 